\title{
Symptom palliation of hypofractionated radiotherapy for patients with incurable inflammatory breast cancer
}

\author{
Hoon Sik Choi ${ }^{1}$, Hong Seok Jang ${ }^{2}$, Ki Mun Kang ${ }^{1,3^{*}}$ and Byung-ock Choi ${ }^{2^{*}}$
}

\begin{abstract}
Background: Incurable inflammatory breast cancer (IBC) patients occasionally suffer from general symptoms such as breast pain, bleeding, ulceration, and discharge, and thus require palliative radiotherapy (RT). Hypofractionated RT has many advantages in palliative settings, but very few studies on IBC have been conducted. This study was conducted to evaluate the effects of hypofractionated RT on symptomatic IBC patients.

Methods: Twenty-two patients with IBC who underwent hypofractionated palliative RT between 2010 and 2016 were retrospectively analyzed. RT was performed at a total dose of 42.5-55 Gy with 2.5-3 Gy per fraction. The treatment effects were evaluated with respect to symptom improvement, tumor response, and treatment-related toxicity.

Results: The main symptoms that the patients complained of before RT were pain, bleeding, and discharge. According to the percentage of symptom relief compared with pre-RT symptoms, the number of patients with $<30,30-70 \%$, and $\geq 70 \%$ were $2(9.1 \%), 7$ (31.8\%), and $13(59.1 \%)$, respectively. Eighteen (81.8\%) patients showed tumor response. No patient experienced grade 3 or higher acute or chronic toxicity during a median follow-up period of 13 months. In univariate analysis, symptom type was a significant factor for predicting the degree of symptom relief. Meanwhile, RT field and C-reactive protein increase were significant factors for predicting the incidence of radiation-induced skin toxicity.
\end{abstract}

Conclusions: Hypofractionated RT could safely and effectively relieve symptoms among incurable symptomatic IBC patients.

Keywords: Inflammatory breast cancer, Palliation, Hypofractionated radiotherapy

\section{Background}

Inflammatory breast cancer (IBC) is a rare disease that accounts for $0.5-2 \%$ of all invasive breast cancers [1]. The standard treatment for non-metastatic IBC is multimodality therapy including neoadjuvant chemotherapy followed by mastectomy and post-mastectomy radiotherapy (RT) [2-4]. However, such aggressive multimodality approach is limited in patients with poor performance status or in

\footnotetext{
*Correspondence: jsk92@gnu.ac.kr; choibo67@catholic.ac.kr

'Department of Radiation Oncology, Gyeongsang National University School of Medicine and Gyeongsang National University Changwon Hospital, 13 Samjungja-ro, Changwon 51472, Republic of Korea

${ }^{2}$ Department of Radiation Oncology, Seoul St. Mary's Hospital, College of Medicine, The Catholic University of Korea, 222, Banpo-daero, Seocho-gu, Seoul, South Korea

Full list of author information is available at the end of the article
}

approximately $30 \%$ of patients with metastatic disease at diagnosis [1]. In such patients, palliative RT is occasionally warranted to control typical symptoms such as breast pain, bleeding, ulceration, and discharge $[5,6]$.

IBC is known for having more radio-resistance than non-IBC; hence, some studies have suggested more aggressive radiation strategies such as increases in the total radiation dose or changes in the fractionation schedules $[7,8]$. In practice, several studies have performed dose escalation with accelerated hyperfractionation schedules in IBC, and these studies have reported an improvement in locoregional control $[3,7,9,10]$. However, this strategy of treating more than twice per day was limited by loading of the treatment machine and accessibility of patients to a hospital. Another altered fractionation 
schedule is hypofractionation; hypofractionated RT delivers a daily dose of $>2$ Gy per fraction and can not only provide good tumor control but also shorten the total treatment period, thereby improving patient convenience and reducing medical expenses [11-13]. However, there have been very few studies on hypofractionated RT for IBC treatment. This might be because the breast skin is affected by inflammatory changes and appears vulnerable to radiation; hence, to date, concerns such as severe toxicities caused by hypofractionated RT conducted by administering large daily doses and involvement of a short overall treatment period remain.

Therefore, it is necessary to analyze the effects of hypofractionated RT on IBC. Here, we retrospectively analyzed the treatment efficacy and toxicities of incurable and symptomatic IBC patients who underwent hypofractionated RT.

\section{Methods}

\section{Patient selection}

Patients who underwent palliative-intent hypofractionated RT for symptomatic IBC between January 2010 and February 2016 at Seoul St. Mary's Hospital, Seoul, were selected for this analysis. Patients with any of the following conditions were included in this study: 1) presence of clinicopathologically proven IBC based on the 8th edition American Joint Committee on Cancer TNM staging system [14, 15], 2) judged medically or surgically incurable as judged by a multidisciplinary team, and 3 ) presence of symptoms such as breast pain, bleeding, ulceration, or discharge requiring urgent symptom palliation. Patients who were initially diagnosed with a metastatic or refractory disease after the initial chemotherapy or hormone therapy were included in this analysis. In contrast, patients with any of the following conditions were excluded: 1) previous history of breast surgery or RT, 2) refusal of RT for personal reasons, and 3) male breast cancer. Thus, a total of 22 IBC patients were eligible for this analysis. Electronic medical records, RT plans, and work-up images were reviewed to analyze treatment effects.

This study was a retrospective, with no informed consent from individual patients, but was performed in accordance with relevant guidelines; the study protocol was approved by the intra-institutional ethics committee of the Seoul St. Mary's Hospital (assigned number: KC15RISI0260).

\section{Hypofractionated RT}

All patients were immobilized with vacuum bags in the arm-up position and computed tomography (CT) scans of their breast were taken. These CT images were imported into Pinnacle treatment planning system, Version 9.1 (Philips Radiation Oncology Systems, Fitchburg, WI, USA). In RT planning, whole breast and skin were not routinely targeted. The principle of target delineation was as follows. The gross lesion responsible for breast symptoms was defined as the gross tumor volume. This volume sometimes included the adjacent metastatic axillary lymph nodes if it was directly connected to the primary tumor. The clinical target volume was not delineated because treatment was delivered with palliative intent. Subsequently, a volumetric margin of $1.0 \mathrm{~cm}$ was applied to create the planning target volume (PTV). If PTV overlapped with the lung or heart, it was manually modified to exclude these organs. Basically, RT plan was designed using $6 \mathrm{MV}$ tangential fields with wedges. Bolus was applied every other day to cover PTV. Two patients for whom the primary mass extended up to the supraclavicular area were also treated with a single anterior oblique photon field. All patients were prescribed a total dose of 42.5-55 Gy with 2.5-3 Gy per fraction, once per day, 5 days a week, for 3-5.5 weeks. In these modest hypofractionated regimens, the total dose was 69.1-89.4 Gy biologically effective dose (BED), which was calculated assuming an alpha/beta ratio of four for breast cancer [16]. The RT plan was normalized such that $100 \%$ of PTV received more than $95 \%$ of the prescribed dose. The ipsilateral mean lung dose was limited to $\leq 20$ Gy with $V_{20 G y}$ of $<$ $30 \%$. The heart was limited to $\mathrm{V}_{35 \mathrm{~Gy}}$ of $<30 \%\left(\mathrm{~V}_{\mathrm{xGy}}\right.$ : the percentage of the organ volume receiving $x$ Gy or more).

\section{Follow-up and statistical analysis}

Before starting RT, all patient symptoms were recorded on a $0-10$ numeric rating scale. During the course of RT, patients were evaluated weekly to assess the degree of symptom relief and treatment-related toxicities. After the end of the RT, patients were generally followed-up at 1 week, then every 2 months for the first year, and then every 6 months for the next 3 years. Symptom relief was quantified by the patient as a percentage of symptom relief compared to their baseline. The degree of symptom relief was divided into three categories by modifying Agarwal et al. study [17] as percentage relief $<30 \%$, percentage relief $30-70 \%$, and percentage relief $>70 \%$. Toxicity was evaluated by the National Cancer Institute's Common Terminology Criteria for Adverse Events (CTCAE) version 4.03. Serum C-reactive protein (CRP) assay was performed before RT, every week during the $\mathrm{RT}$, and then upon follow-up to estimate the degree of skin irritation through increased systemic inflammation marker $[18,19]$. CT was performed at 2 months after $\mathrm{RT}$, and every 3 months afterward for treatment response evaluation. The response was evaluated by the Response Evaluation Criteria in Solid Tumors (RECIST) criteria. Overall survival (OS) duration was defined as the period from the end date of RT to the date of death for any reason. OS analysis was performed using the Kaplan-Meier method. The relations between patient, 
tumor, and RT characteristics with treatment outcomes (symptom relief and toxicity) were evaluated by univariate analysis using logistic regression. Symptom relief was analyzed based on 1-month results after the end of RT when the most clinically symptomatic improvement is expected. Treatment-related toxicity was analyzed based on all adverse events occurring during the follow-up period. All analyses were performed using the SPSS program (Version 21.0; SPSS, Inc., Chicago, IL, USA). $P$ values $<0.05$ were considered statistically significant.

\section{Results}

\section{Patient characteristics}

A total of 22 patients were included in this study. Table 1 provides a summary of the patients' characteristics. Their median age at the time of RT was 66 years (range: $39-$ 90 years). The Eastern Cooperative Oncology Group performance score for most (90.9\%) of these patients was 0 or 1. According to the 8th TNM staging system, the number of patients with N0, N1, N2, and N3 was 1 (4.5\%), 1 (4.5\%), 11 (50\%), and 9 (40.9\%), respectively. Regarding M stage, 10 (45.5\%) patients were in the M0 stage, while $12(54.5 \%)$ were in the M1 stage. Nine (40.9\%) patients had received chemotherapy or hormone therapy before RT, but the disease continued to progress. Regarding RT technique, the prescribed dose was $<80$ Gy (BED, alpha/beta ratio $=4)$ for $31.8 \%(7 / 22)$ of patients and $\geq 80$ Gy $(B E D$, alpha/beta ratio $=4)$ for $68.2 \%$ $(15 / 22)$ of the patients.

After approximately 1 month of RT, palliative chemotherapy or hormone therapy was performed in $77.3 \%$ $(17 / 22)$ of patients. The median follow-up duration was 13.9 months (range, 1.1-81.2 months), and six patients

Table 1 Patient characteristics

\begin{tabular}{lll}
\hline Characteristic & & Number of patients \\
\hline Age (years) & 0 or 1 & $20(90.9 \%)$ \\
ECOG PS & 2 & $2(9.1 \%)$ \\
Pathology & IDC & $20(90.9 \%)$ \\
& IMC & $2(9.1 \%)$ \\
N stage & 0 or 1 & $2(9.1 \%)$ \\
& 2 or 3 & $20(90.9 \%)$ \\
M stage & 0 & $10(45.5 \%)$ \\
Previous CTx or HTx history & 1 & $12(54.5 \%)$ \\
Yes & $9(40.9 \%)$ \\
Prescribed dose (BED) & No & $13(59.1 \%)$ \\
& $70-80$ Gy & $7(31.8 \%)$ \\
\hline ECOG Eang)
\end{tabular}

ECOG Eastern Cooperative Oncology Group, PS performance status, IDC invasive ductal carcinoma, IMC invasive medullary carcinoma, CTX chemotherapy, HTx hormone therapy, BED biologically effective dose were alive at the end of the follow-up period. The median OS was 17.3 months, and 1-year and 2-year OS rates were 62.8 and $34.5 \%$, respectively.

\section{Symptom relief and tumor response}

The main symptoms requiring palliative RT were pain (13 patients, 59.1\%), bleeding (4 patients, 18.2\%), and discharge (13 patients, 59.1\%). Eight patients had two or more combination of symptoms. Stratification for symptom relief $(<30$, $30-70 \%$, and $\geq 70 \%$ ) was performed according to the percentage of symptom relief in comparison with the initial symptoms, and the results are listed in Table 2 . In total, the number of patients with $<30,30-70 \%$, and $\geq 70 \%$ symptom relief were 2 (9.1\%), 7 (31.8\%), and $13(59.1 \%)$, respectively. Among the main symptoms, pain relief tended to be more prominent than decrease in bleeding or discharge. Two patients who had symptom relief of $<30 \%$ after RT subsequently received palliative chemotherapy, but the symptoms continued to deteriorate rapidly and the patients died shortly afterward. After evaluating the response according to RECIST criteria, 18 (81.8\%) patients showed a partial response, three (13.6\%) patients showed stable disease, and the remaining one $(4.5 \%)$ patient showed progressive disease during the follow-up period. Table 3 shows the univariate analysis of patient characteristics on the degree of symptom relief. In the univariate analysis, symptom type $(p=0.02)$ was a significant clinical factor for predicting the degree of symptom relief. Patients with a single symptom had significantly better symptom relief than those with overlapping symptoms. In addition, although not statistically significant, symptom relief tended to be better in patients who received a high radiation dose (BED $\geq 80 \mathrm{~Gy}, \mathrm{a} / \mathrm{b}$ ratio $=4, p=0.06$ ).

\section{Toxicity}

Treatment was well-tolerated. Based on CTCAE criteria, acute complications during and shortly after RT were grade 1 skin dermatitis (faint erythema and dry desquamation) in six patients (27.3\%) and grade 2 skin dermatitis (moist desquamation) in 16 patients (72.7\%). No patient developed grade 3 or higher skin toxicity and none died from treatment-related toxicity. Chronic complications of radiation, severe chest wall fibrosis, rib fracture, arm edema, and symptomatic pneumonitis were not

Table 2 Degree of symptom relief at 1 week after the end of radiotherapy

\begin{tabular}{llll}
\hline & \multicolumn{3}{l}{ Degree of symptom relief compared to baseline } \\
\cline { 2 - 4 } & $<30 \%$ & $30-70 \%$ & $\geq 70 \%$ \\
\hline Pain $(n=13)$ & $1(7.7 \%)$ & $3(23.1 \%)$ & $9(69.2 \%)$ \\
Bleeding $(n=4)$ & 0 & $3(75 \%)$ & $1(25 \%)$ \\
Discharge $(n=13)$ & $2(15.4 \%)$ & $7(53.8 \%)$ & $4(30.8 \%)$ \\
Total $(n=22)$ & $2(9.1 \%)$ & $7(31.8 \%)$ & $13(59.1 \%)$ \\
\hline
\end{tabular}


Table 3 Univariate analysis of the degree of symptom relief

\begin{tabular}{llll}
\hline Variables & HR & $95 \% \mathrm{Cl}$ & $p$ value \\
\hline Pathology (IDC vs. IMC) & 0.36 & $0.06-2.23$ & 0.27 \\
Previous CTx or HTx history (Yes vs. No) & 0.68 & $0.14-3.25$ & 0.63 \\
Prescription dose (<80 Gy ${ }_{4}$ vs. $\geq 80$ Gy ${ }_{4}$ ) & 0.70 & $0.05-5.12$ & 0.06 \\
Symptom type (single vs. combination) & 3.57 & $1.87-49.08$ & 0.02 \\
Tumor response (PR vs. SD + PD) & 0.16 & $0.01-1.84$ & 0.14
\end{tabular}

$H R$ hazard ratio, $C I$ confidential interval, IDC invasive ductal carcinoma, IMC invasive medullary carcinoma, CTx chemotherapy, HTx hormone therapy, Gy biologically effective dose of alpha/beta ratio $4, P R$ partial response, $S D$ stable disease, $P D$ progressive disease

observed in any patient. These side effects in patients were improved by appropriate supportive care such as frequent dressing, application of topical agents, and collaboration with a dermatologist when necessary. To quantitatively analyze the degree of skin dermatitis, changes in CRP values before, during, and after the RT period were measured. The normal CRP value was $0-5 \mathrm{mg} / \mathrm{L}$. In four patients $(18.2 \%)$, the CRP level gradually increased during the RT period and fell to the normal range 1 month after RT. The median CRP values before ( 1 week before $\mathrm{RT}$ ), during ( 2 weeks during $\mathrm{RT})$, and after the RT period (1 month after RT) were 6.3, 5.9 , and $2.5 \mathrm{mg} / \mathrm{L}$, respectively. Figure 1 shows the change in CRP median values through box-and-whisker plots. Table 4 shows the univariate analysis of patient characteristics on skin toxicity according to CTCAE. In the univariate analysis, the RT field $(p=0.04)$ and CRP increase during the RT period $(p=0.03)$ were significant factors for predicting the incidence of radiation-induced skin toxicity.

\section{Discussion}

We retrospectively analyzed 22 patients who received hypofractionated RT for symptomatic IBC. Most patients showed symptom relief with acceptable skin toxicity. Although OS was much poorer than that of patients who underwent curative-intent standard multimodality
Table 4 Univariate analysis of skin toxicity according to CTCAE

\begin{tabular}{llll}
\hline Variables & HR & $95 \% \mathrm{Cl}$ & $p$ value \\
\hline Age (<65 years vs. $\geq 65$ years) & 0.76 & $0.04-3.21$ & 0.49 \\
Previous CTx or HTx history (Yes vs. No) & 1.21 & $0.43-4.24$ & 0.55 \\
RT fraction size ( 2.5 Gy vs. > 2.5 Gy) & 0.89 & $0.01-2.05$ & 0.18 \\
Prescription dose (< 80 Gy $_{4}$ vs. $\left.\geq 80 \mathrm{~Gy}_{4}\right)$ & 0.92 & $0.31-6.17$ & 0.25 \\
RT field (breast mass vs. breast $+\mathrm{SCL})$ & 0.86 & $0.01-5.45$ & 0.04
\end{tabular}

CRP increase during the RT period (Yes vs. No) $2.52 \quad 1.01-8.92 \quad 0.03$

$H R$ hazard ratio, $C l$ confidential interval, CTx chemotherapy, HTx hormone therapy, $R T$ radiotherapy, Gy 4 biologically effective dose of alpha/beta ratio 4 , $S C L$ supraclaviculr lymph node, CRP C-reactive protein

treatment, $81.8 \%$ of patients showed a tumor response within the follow-up period.

It is uncommon to encounter unresectable breast cancer patients in this era when screening tools have been developed and neoadjuvant chemotherapy can be performed. Despite not being tumor resected, RT still plays an important role in women suffering from ulceration, bleeding, or pain in locally advanced and recurrent breast cancer [20, 21]. Although there are no clear guidelines for the most appropriate palliative RT dose and fractionation schedules, tumor control tends to increase with increasing total radiation dose [22]. In addition, hypofractionated schedules were often used in palliative RT to improve patient inconvenience by reducing treatment duration [23-25]. Based on this knowledge, hypofractionated palliative RT of 42.5-55 Gy with 2.5-3 Gy per fraction was performed in our study to increase the RT effect. This schedule was similar to the dose schedules used in hypofractionated whole breast RT studies for adjuvant aim after breast conservation surgery, and long-term results of these studies have revealed that this schedule has a safe outcome [13, 26, 27].

To date, no study has focused on hypofractionated palliative RT of IBC patients; hence, it is difficult to

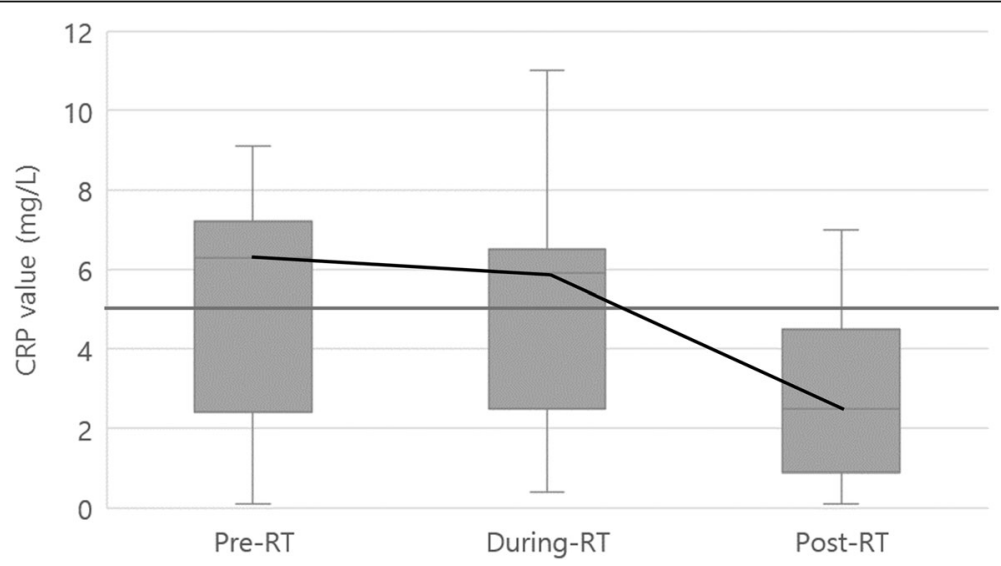

Fig. 1 Box-and-whisker plot of C-reactive protein level according to time based on radiotherapy. The straight gray line is the normal value (0-5 $\mathrm{mg} / \mathrm{L}$ ) drawn for reference, and the black trend line shows the change in the median value. CRP, C-reactive protein; RT, radiotherapy 
undertake a direct comparison of the palliation effect. However, indirect comparisons with existing non-IBC studies are possible. Richard et al. [5] reported the effect of palliative RT on 13 locally advanced breast cancer patients with skin ulceration. The median dose was 27.54 Gy in 11 fractions, and $46.2 \%$ of patients showed clinical improvement. Only doses that exceed 30 Gy were found to be effect in patients. Nakamura et al. [28] performed a prospective study to evaluate the effectiveness of various palliative RT regimes for 21 patients with breast cancer including skin invasion; most of their patients received 36 Gy in 12 fractions, but some patients received a relatively high-dose regimen such as 50 Gy in 20 fractions or $60 \mathrm{~Gy}$ in 30 fractions. In a majority of the patients in that study, bleeding, discharge, and offensive odor reduced and the quality of life score improved. However, they suggested that dose fractionation optimization is necessary because symptoms tended to re-progress after approximately 6 months. In our study, approximately $60 \%$ of patients reported $\geq 70 \%$ symptom improvement in comparison with pre-RT symptoms, and these improvements were marked by a single symptomatic pain. Although not statistically significant, symptomatic improvement was detected at dosed greater than BED $80 \mathrm{~Gy}(\mathrm{a} / \mathrm{b}$ ratio $=4)$.

RT-related toxicity was influenced by both fraction size and a wide variety of factors. Bristol et al. reported 10$20 \%$ grade 3 or higher treatment-related toxicity in IBC patients receiving curative-intent aggressive multimodality treatment [3]. In contrast, Whelan et al. reported < $3 \%$ toxicity of grade 3 or higher in patients with early breast cancer who underwent hypofractionated RT following breast-conserving surgery [13]. In our study, neither late toxicity within the follow-up period nor acute toxicity of grade 3 or higher was observed. The difference in toxicity between these studies was considered to be due to the RT field, which was considered to be a significant factor affecting toxicity and extent of surgery in our study. We believe that the toxicity was low in our study because surgery was not performed in all the patients, and irradiation was limited to the tumor mass and did not frequently include the regional lymphatic areas. Our study also revealed that the CRP level did not increase with RT in most patients. Of course, the CRP level not only reflects inflammation of the skin but also of the whole body; the CRP level can change for various reasons including bacterial superinfection and cancer progression. However, some studies have reported a causal relationship between skin dermatitis and CRP levels $[19,29]$. To minimize CRP level variation, we performed repeated measurements of CRP levels at 1-week intervals. In addition, we examined the use of antibiotics to influence the CRP level and confirmed that no antibiotic was used in any patients during the RT period. Patients with increased CRP levels were more likely to develop skin toxicity; hence, active supportive care can be applied according to CRP level changes.

This study has several limitations. The first was the retrospective nature of the study, which may have resulted in heterogeneous patient characteristics particularly among $40.9 \%$ of patients who had received previous chemotherapy or hormone therapy, and the second was the relatively small number of cancer patients. However, our study is meaningful in that it focused on patients with IBC as well as on the effects of hypofractionated palliative RT. In future, further studies with more patients will be required to confirm the effect of hypofractionated palliative RT on the quality of life of patients or on complications seen later during the long-term followup. In addition, it is possible to conduct studies to examine the increase in local control and disease-free survival by adding aggressive treatment to patients who respond to hypofractionated RT.

\section{Conclusions}

Our results suggest that hypofractionated RT was relatively effective fo the mono-symptom of breast pain in incurable and symptomatic IBC patients. In addition, this treatment was safe and well tolerated with acceptable toxicity. Further studies to determine an appropriate RT scheme, such as dose fractionation schedule and RT field, are warranted to balance the effects and toxicity.

\section{Abbreviations \\ BED: Biologically effective dose; CRP: C-reactive protein; CT: Computed tomography; CTCAE: Common Terminology Criteria for Adverse Events; IBC: Inflammatory breast cancer; OS: Overall survival; PTV: Planning target volume; RECIST: Response Evaluation Criteria In Solid Tumors; \\ RT: Radiotherapy}

\section{Acknowledgements}

Not applicable.

\section{Authors' contributions}

Conception and design of the study: HSC, HSJ, and BC. Acquisition of data: HSJ, and BC. Analysis and interpretation of the data: HSC, and KMK. Writing and revision of the manuscript: HSC, HSJ, and BC. All authors read and approved the final manuscript.

\section{Funding}

This study was not funded by any organizations or companies.

\section{Availability of data and materials}

Raw data may be available on request from the corresponding author.

\section{Ethics approval and consent to participate}

This study was a retrospective study with no informed consent from the individual patient, but was performed according to the relevant guidelines and protocol approved by the intra-institutional ethics committee of the Seoul St. Mary's hospital (assigned number: KC15RISI0260).

\section{Consent for publication}

Not applicable because this analysis has retrospective nature.

Competing interests

The authors declare that they have no competing interests. 


\section{Author details}

'Department of Radiation Oncology, Gyeongsang National University School of Medicine and Gyeongsang National University Changwon Hospital, 13 Samjungja-ro, Changwon 51472, Republic of Korea. ${ }^{2}$ Department of Radiation Oncology, Seoul St. Mary's Hospital, College of Medicine, The Catholic University of Korea, 222, Banpo-daero, Seocho-gu, Seoul, South Korea. ${ }^{3}$ Institute of Health Science, Gyeongsang National University, Jinju, Republic of Korea.

Received: 12 March 2019 Accepted: 11 June 2019

Published online: 20 June 2019

\section{References}

1. Wingo PA, Jamison PM, Young JL, Gargiullo P. Population-based statistics for women diagnosed with inflammatory breast cancer (United States). Cancer Causes Control. 2004;15:321-8.

2. Abramowitz MC, Li T, Morrow M, Sigurdson ER, Anderson P, Nicolaou N, Freedman G. Dermal lymphatic invasion and inflammatory breast cancer are independent predictors of outcome after postmastectomy radiation. Am J Clin Oncol. 2009:32:30-3.

3. Bristol IJ, Woodward WA, Strom EA, Cristofanilli M, Domain D, Singletary SE, Perkins GH, Oh JL, Yu TK, Terrefe W, et al. Locoregional treatment outcomes after multimodality management of inflammatory breast cancer. Int J Radiat Oncol Biol Phys. 2008:72:474-84.

4. Brown L, Harmsen W, Blanchard M, Goetz M, Jakub J, Mutter R, Petersen I, Rooney J, Stauder M, Yan E, et al. Once-daily radiation therapy for inflammatory breast cancer. Int J Radiat Oncol Biol Phys. 2014;89:997-1003.

5. Vempati P, Knoll MA, Dharmarajan K, Green S, Tiersten A, Bakst RL. Palliation of ulcerative breast lesions with radiation. Anticancer Res. 2016;36:4701-5.

6. Matro JM, Li T, Cristofanilli M, Hughes ME, Ottesen RA, Weeks JC, Wong YN. Inflammatory breast cancer management in the national comprehensive cancer network: the disease, recurrence pattern, and outcome. Clin Breast Cancer. 2015:15:1-7.

7. Liao Z, Strom EA, Buzdar AU, Singletary SE, Hunt K, Allen PK, McNeese MD. Locoregional irradiation for inflammatory breast cancer: effectiveness of dose escalation in decreasing recurrence. Int J Radiat Oncol Biol Phys. 2000;47:1191-200.

8. Plataniotis GA, Dale RG. Biologically effective dose-response relationship for breast cancer treated by conservative surgery and postoperative radiotherapy. Int J Radiat Oncol Biol Phys. 2009;75:512-7.

9. Gurney H, Harnett P, Kefford R, Boyages J. Inflammatory breast cancer: enhanced local control with hyperfractionated radiotherapy and infusional vincristine, ifosfamide and epirubicin. Aust NZ J Med. 1998;28:400-2

10. Pisansky TM, Schaid DJ, Loprinzi CL, Donohue JH, Schray MF, Schomberg PJ. Inflammatory breast cancer: integration of irradiation, surgery, and chemotherapy. Am J Clin Oncol. 1992;15:376-87.

11. Haviland JS, Owen JR, Dewar JA, Agrawal RK, Barrett J, Barrett-Lee PJ, Dobbs HJ, Hopwood P, Lawton PA, Magee BJ, et al. The UK standardisation of breast radiotherapy (START) trials of radiotherapy hypofractionation for treatment of early breast cancer: 10-year follow-up results of two randomised controlled trials. Lancet Oncol. 2013;14:1086-94.

12. Owen JR, Ashton A, Bliss JM, Homewood J, Harper C, Hanson J, Haviland J, Bentzen SM, Yarnold JR. Effect of radiotherapy fraction size on tumour control in patients with early-stage breast cancer after local tumour excision: longterm results of a randomised trial. Lancet Oncol. 2006;7:467-71.

13. Whelan TJ, Pignol JP, Levine MN, Julian JA, MacKenzie R, Parpia S, Shelley W, Grimard L, Bowen J, Lukka H, et al. Long-term results of hypofractionated radiation therapy for breast cancer. N Engl J Med. 2010;362:513-20.

14. Dawood S, Merajver SD, Viens P, Vermeulen PB, Swain SM, Buchholz TA, Dirix LY, Levine PH, Lucci A, Krishnamurthy $S$, et al. International expert panel on inflammatory breast cancer: consensus statement for standardized diagnosis and treatment. Ann Oncol. 2011;22:515-23.

15. Quinn C, Rakha EA. Eighth edition Cancer staging manual of breast Cancer by the American joint committee on Cancer: are the new changes to improve staging or a treatment decision tool? J Clin Pathol. 2018;71:1028-9.

16. Fowler JF. The linear-quadratic formula and progress in fractionated radiotherapy. Br J Radiol. 1989:62:679-94.

17. Agarwal JP, Nemade B, Murthy V, Ghosh-Laskar S, Budrukkar A, Gupta T, D'Cruz A, Pai P, Chaturvedi P, Dinshaw K. Hypofractionated, palliative radiotherapy for advanced head and neck cancer. Radiother Oncol. 2008;89:51-6.
18. Landry A, Docherty P, Ouellette $S$, Cartier $\sqcup$. Causes and outcomes of markedly elevated C-reactive protein levels. Can Fam Physician. 2017;63:e316-e23.

19. Hu JJ, Urbanic JJ, Case LD, Takita C, Wright JL, Brown DR, Langefeld $C D$, Lively MO, Mitchell SE, Thakrar A, et al. Association between inflammatory biomarker $\mathrm{C}$-reactive protein and radiotherapy-induced early adverse skin reactions in a multiracial/ethnic breast Cancer population. J Clin Oncol. 2018;36:2473-82.

20. Bedwinek JM, Lee J, Fineberg B, Ocwieza M. Prognostic indicators in patients with isolated local-regional recurrence of breast cancer. Cancer. 1981;47:2232-5.

21. Price A, Kerr GR, Rodger A. Primary radiotherapy for T4 breast cancer. Clin Oncol (R Coll Radiol). 1992;4:217-21.

22. Lutz ST, Jones J, Chow E. Role of radiation therapy in palliative care of the patient with cancer. J Clin Oncol. 2014;32:2913-9.

23. Das S, Thomas S, Pal SK, Isiah R, John S. Hypofractionated palliative radiotherapy in locally advanced inoperable head and neck Cancer: CMC Vellore experience. Indian J Palliat Care. 2013;19:93-8.

24. Duchesne GM, Bolger JJ, Griffiths GO, Trevor Roberts J, Graham JD, Hoskin PJ, Fossa SD, Uscinska BM, Parmar MK. A randomized trial of hypofractionated schedules of palliative radiotherapy in the management of bladder carcinoma: results of medical research council trial BA09. Int J Radiat Oncol Biol Phys. 2000;47:379-88.

25. Sundstrom S, Bremnes R, Aasebo U, Aamdal S, Hatlevoll R, Brunsvig P, Johannessen DC, Klepp O, Fayers PM, Kaasa S. Hypofractionated palliative radiotherapy (17 Gy per two fractions) in advanced non-small-cell lung carcinoma is comparable to standard fractionation for symptom control and survival: a national phase III trial. J Clin Oncol. 2004;22:801-10.

26. Kim KS, Shin KH, Choi N, Lee SW. Hypofractionated whole breast irradiation: new standard in early breast cancer after breast-conserving surgery. Radiat Oncol J. 2016;34:81-7.

27. Deantonio L, Cozzi S, Tunesi S, Brambilla M, Masini L, Pisani C, Gambaro G, Magnani C, Krengli M. Hypofractionated radiation therapy for breast cancer: long-term results in a series of 85 patients. Tumori. 2016;102:398-403.

28. Nakamura N, Kawamori J, Takahashi O, Shikama N, Sekiguchi K, Takahashi T, Kato S, Ogita M, Motegi A, Akimoto T. Palliative radiotherapy for breast cancer patients with skin invasion: a multi-institutional prospective observational study. Jpn J Clin Oncol. 2018;48:555-8.

29. Vekaria AS, Brunner PM, Aleisa Al, Bonomo L, Lebwohl MG, Israel A, Guttman-Yassky E. Moderate-to-severe atopic dermatitis patients show increases in serum C-reactive protein levels, correlating with skin disease activity. F1000Res. 2017;6:1712.

\section{Publisher's Note}

Springer Nature remains neutral with regard to jurisdictional claims in published maps and institutional affiliations.

\section{Ready to submit your research? Choose BMC and benefit from:}

- fast, convenient online submission

- thorough peer review by experienced researchers in your field

- rapid publication on acceptance

- support for research data, including large and complex data types

- gold Open Access which fosters wider collaboration and increased citations

- maximum visibility for your research: over $100 \mathrm{M}$ website views per year

At BMC, research is always in progress.

Learn more biomedcentral.com/submissions 